\title{
Influencia de los Estilos de Liderazgo y las Prácticas de Gestión de RRHH sobre el Clima Organizacional de Innovación
}

\section{Influence of Leadership Styles and Human Resources Management Practices on Innovation Climate in Organizations}

\author{
Fernando J. Pons Verdú y José Ramos López \\ Universitat de València
}

\begin{abstract}
Resumen. Frente a propuestas de valor asociadas a un bajo coste productivo, numerosas naciones y organizaciones buscan convertir a la innovación en la palanca de un nuevo modelo de crecimiento económico. Por su marcado carácter social, este estudio analiza la relación entre el clima organizacional de innovación, los estilos de liderazgo y las prácticas de gestión de RRHH características en la organización. Los modelos de regresión obtenidos explican una varianza cercana al $60 \%$ del clima organizacional innovador y señalan a las prácticas dirigidas a estimular la participación de los miembros y el estilo de liderazgo inspiracional como sus principales predictores.
\end{abstract}

Palabras clave: clima organizacional de innovación, estilos de liderazgo, prácticas de gestión de RRHH.

\begin{abstract}
An increasing number of organizations and countries are focusing in innovations as the lever for a new model of economic development, front proposals based in decreasing costs. Due to their marked social character, the present study analyses the relationship between organizational climate of innovation and the leadership styles and practices of human resources management addressed by organizations to their members. Regression analyses carried out explained near of $60 \%$ of the variance in innovation climate, outstanding HRM practices addressed to stimulate workers participation and inspirational leadership as main predictors.

Keywords: innovation climate, leadership, human resources management practices.
\end{abstract}

\section{Introducción}

Los cambios socioeconómicos están provocando que numerosos países traten de generar estrategias de promoción de la innovación para asegurar su competitividad frente a países de bajos costes productivos. De hecho, la actividad innovadora constituye, junto al capital humano, uno de los principales factores que determinan las ventajas competitivas de las economías avanzadas (Chesnais, 1990). El papel que la innovación está jugando en la supervivencia de las organizaciones ha hecho que el interés por su comprensión haya crecido, tanto en el ámbito científico como en el profesional (Ford y Gioia, 1995; King y Anderson, 2002). Este cambio en la naturaleza del trabajo en las organizaciones ha motivado que los estudios sobre la innovación hayan crecido en las últimas décadas (Amabile, 1983; Anderson, De Dreu y Nijstad, 2004; Van de Ven, Angle y Poole, 1989; West, 2002).

Por su naturaleza social, la innovación no puede

La correspondencia sobre este artículo deberá ser dirigida a José Ramos. Facultad de Psicología. Av. Blasco Ibáñez, 21. 46010 Valencia. E-mail:ramosj@uv.es. desarrollarse en el vacío. Los elementos creativos, necesarios para el desarrollo de las nuevas ideas, implican cierto nivel de interacción social antes de que puedan ser tratados como innovaciones. Es en este sentido en el que aspectos contextuales como el clima organizacional cobran importancia. Aunque por lo general la investigación ha considerado el clima global de la organización (Kuenzi y Schminke, 2009), algunos estudios se han centrado en facetas particulares, como el clima para la innovación (Anderson y West, 1992; Eisenbeiss, van Knippenberg y Boerner, 2008; Scott y Bruce, 1994). Un clima de innovación estimula a los empleados a contribuir de manera creativa a la misión de la organización. Conocer sus antecedentes organizacionales puede ayudar a diseñar un contexto más sugerente para el desarrollo de la capacidad innovadora de la organización.

Las organizaciones necesitan ampliar el ámbito de actuación sobre sus miembros, más allá de la esfera de lo observable. Necesitan influir positivamente en las actitudes de sus miembros para generar una movilización voluntaria hacia los objetivos organizacionales. Existen diversas formas mediante las que la organización puede influir sobre sus miembros. Por ejemplo, a 
través de la forma en que dirige y gestiona a sus Recursos Humanos. La relación entre el clima de innovación, los estilos de liderazgo y las prácticas de gestión de RR.HH. ha sido objeto de estudio en investigaciones recientes (p.ej., Charbonnier-Voirin, El Akremi, y Vandenberghe, 2010; Chang, Gong y Shum, 2011). El estilo de liderazgo ejercido por el superior y las prácticas de gestión de RR.HH. destacan sobre otros aspectos organizacionales por la proximidad en la que los empleados perciben sus efectos. Cómo se comporta el inmediato superior de un trabajador y su percepción sobre cómo le trata la empresa, son dos aspectos a considerar si se quiere analizar la forma de actuar sobre la percepción del clima laboral.

\section{Clima de innovación}

Tradicionalmente el concepto de clima organizacional ha sido confuso y se ha definido de múltiples formas. Básicamente existen dos aproximaciones contrarias en cuanto a su naturaleza (Baer y Frese, 2003). Por un lado, algunos autores entienden el clima organizacional como un agregado de climas psicológicos individuales. De este modo, cada clima psicológico sería el resultado de la interpretación cognitiva que cada miembro realiza sobre una determinada situación organizacional. (James, James y Ashe, 1990). Desde esta teoría, se sugiere que los individuos se comportan principalmente en función de las representaciones cognitivas que tienen del ambiente, "en lugar de por cómo es el ambiente en sî" (James y Sells, 1981). Por lo tanto, el clima representaría las señales que reciben los individuos acerca de las expectativas de la organización sobre su conducta y sus potenciales consecuencias. Así, los empleados pueden usar esta información para generar sus expectativas y sus creencias (James, Hartman, Stebbins y Jones, 1977). Del mismo modo, las personas responden a estas expectativas regulando su conducta, con objeto de realizar una autoevaluación positiva y esperar unas consecuencias también positivas, como el orgullo y la autosatisfacción (Bandura, 1988)

Una segunda aproximación se refiere a la conceptualización del clima como un atributo de la organización más que del individuo, que lo entiende como el resultado de procesos sociológicos y organizacionales (Glick, 1988). Desde esta posición se define el clima organizacional como "un término genérico que integra variables organizacionales y psicológicas, que describe el contexto organizacional y condiciona las acciones de los individuos" (Glick, 1985). Una definición más reciente de Isaksen, Lauer, Ekval y Britz (2000-2001) describe el clima como las "actitudes, sentimientos y patrones de comportamiento recurrentes y observables que caracterizan la vida en la organización”. Según el nivel de análisis, el concepto de clima puede ser dividido en dos constructos distintos pero complementa- rios: clima psicológico y organizacional. Esto es, el clima organizacional describe la vida en la organización y depende de climas psicológicos agregados.

En el ámbito de la creatividad y la innovación, Amabile afirma que estas percepciones, más que el propio ambiente objetivo, tienen una influencia crucial sobre la motivación y la creatividad de los empleados (Amabile y Gryskiewicz, 1989; Amabile, Taylor y Gryskiewicz, 1995). No obstante, según Kwasniewska y Necka (2004), el impacto del clima sobre la creatividad es distinto según el puesto ocupado, siendo superior en aquellas personas que ocupan un puesto de dirección en la organización. Así, mientras el clima se ha definido frecuentemente como "las percepciones de los empleados sobre sus organizaciones", el constructo ha sufrido durante años definiciones contrarias e inconsistentes en su operacionalización. Hasta ahora, la aproximación más aceptada al concepto de clima lo define como "las percepciones compartidas de los empleados sobre los acontecimientos, prácticas y procedimientos de la organización" (Patterson et al., 2005).

Schneider (1975) sugirió que hay muchos tipos de clima, y Schneider y Reichers señalaron que "hablar de clima organizacional per se, sin relacionarlo con algún referente, no tiene sentido" (1983). Una asunción inicial de las teorías sobre el clima organizacional era que los ambientes sociales podrían estar caracterizados por un número limitado de dimensiones. A partir de esta premisa, la proliferación del número de dimensiones identificadas ha producido cierto grado de confusión y ha dificultado el progreso teórico (Patterson et al., 2005). Respecto a su evaluación, Schneider (1975, 1990; 2000) evita el empleo de medidas multidimensionales del clima. Este autor sugiere que las dimensiones del clima organizacional se diferencian según el objetivo de la investigación y que las medidas generales contendrán dimensiones que no son relevantes para cada estudio específico. Por este motivo, propone la medida del clima específico que interesa relacionar con el resto de variables de la investigación. Esta línea argumental ha animado el desarrollo de medidas de distintas dimensiones de clima, como la orientación al servicio (Schneider, 1990) y la innovación (Anderson y West, 1992; West, 1990). Por otro lado, además del nivel organizacional que se ha utilizado en este trabajo, el clima puede referirse a unidades menores dentro de la organización como un grupo o un equipo de trabajo. En el ámbito de la innovación, el estudio empírico de los efectos del clima sobre la conducta innovadora ha sido limitado (Scott y Bruce, 1994), habiéndose encontrando más evidencias de la relación con la innovación en el nivel grupal (p.ej., De Dreu y de Vries, 1997; De Dreu y West, 2001; Tjosvold, 1998; West y Anderson, 1996).

Algunos investigadores han señalado que las organizaciones innovadoras se caracterizan por estar orientadas hacia la creatividad y el cambio, por apoyar la 
independencia de sus empleados en la búsqueda de nuevas ideas (Kanter, 1983; Siegel y Kaemmerer, 1978) y gestionar adecuadamente la diversidad de sus miembros (Siegel y Kaemmerer, 1978). Por otro lado, que la organización facilite los recursos y el tiempo necesario, también se ha mostrado como un factor crítico para desarrollar la innovación (Amabile, 1988; Angle, 1989).

En el ámbito de las innovaciones de proceso a nivel organizacional, el éxito y la efectividad de los cambios depende del esfuerzo global de la organización. Las organizaciones que presentan un ambiente de trabajo caracterizado por la iniciativa y la seguridad psicológica muestran una probabilidad mayor de que sus innovaciones sean implementadas eficazmente (Baer y Frese, 2003). Estos autores plantean que el clima organizacional es un factor contingente que condiciona la efectividad de las prácticas organizacionales y la implementación de las innovaciones de proceso (p.ej. en la gestión de la calidad total). En consecuencia, señalan la necesidad de desarrollar un clima en el que las personas se sientan seguras cuando tengan que asumir riesgos, proponer nuevas ideas o hablar abiertamente de los problemas. De no ser así, el proceso de implementación de la innovación puede acabar siendo un fracaso. A nivel individual, además de la relación entre el clima de innovación y actitudes laborales como el compromiso, en las últimas décadas también se ha analizado su relación con distintos niveles de comportamiento, como las conductas de innovación y colaboración (Mañas, González y Peiró, 1999). Así, diversas investigaciones en el ámbito de la asistencia médica y la dirección de equipos sugieren que el clima grupal de innovación influye en el comportamiento innovador de sus miembros (West y Anderson, 1996; West y Wallace, 1991).

En resumen, tanto los estudios a nivel organizacional como los centrados en niveles inferiores (grupal e individual) han ofrecido un apoyo empírico a los efectos del clima sobre la innovación (Abbey y Dickson, 1983; Paolillo y Brown, 1978; Siegel y Kaemmerer, 1978).

\section{Estilo de liderazgo}

Un empleado innovador depende de su superior para tener acceso a la información (datos, know how, políticas internas, etc.), los recursos (materiales, espacio, tiempo, etc.) y el apoyo sociopolítico (aprobación, legitimidad, apoyo, etc.) necesario para desarrollar y poner en práctica la innovación (Janssen, De Vliert y West, 2004). El trabajo creativo e innovador es ambiguo, arriesgado y sujeto a críticas. Por este motivo, contar con el apoyo del superior inmediato puede facilitar la creatividad, mientras que una supervisión más crítica y con mayor énfasis en el control puede tender a inhibirla (Amabile y Gryskiewicz, 1989; West y Farr, 1989). Según Janssen (2005), cuando el sujeto se sien- te apoyado por su superior se incrementa la percepción de su capacidad para influir de manera innovadora en el ambiente de trabajo. Este hallazgo sugiere que los empleados sienten que sus supervisores son actores clave, ya que tienen el poder de prestar o negar el apoyo necesario para el desarrollo de las nuevas ideas. Asimismo, apunta a una relación entre el estilo de liderazgo y el clima de innovación. De manera que un estilo de liderazgo que favorezca la innovación puede influir en la percepción del clima de innovación en la organización.

En otra investigación Axtell et al. (2000) encontraron que la eficacia de la puesta en práctica de las ideas innovadoras de los empleados dependía del estilo de apoyo de su supervisor. Desde la teoría de las metas de logro se sugiere que el estilo adoptado por el supervisor puede influir en la forma en la que se aproxima, interpreta y responde a las ideas innovadoras expresadas por sus colaboradores (Jansen et al., 2004). Jansen, Schoonebeek y van Looy (1997) encontraron que el liderazgo considerado y consultivo correlacionaba con el empowerment del empleado, que a su vez, lo hacía con el comportamiento innovador.

En otro estudio sobre la relación entre el estilo de liderazgo y la efectividad en equipos de $\mathrm{I}+\mathrm{D}$, los resultados sugerían claramente que el estilo de liderazgo es importante para la eficacia en la gestión del equipo (Stoker, Looise, Fisscher y de Jong, 2001). En este estudio se analizó la relación entre los estilos de liderazgo carismático y participativo de los responsables del equipo y, la satisfacción laboral, el compromiso organizacional y la eficacia grupal percibida por los miembros del grupo. Los resultados mostraron una correlación positiva entre los dos estilos de liderazgo y el resto de las variables, siendo especialmente significativa entre el estilo carismático y las variables de resultados.

Entre los factores ambientales específicos, el liderazgo carismático parece facilitar en menor medida la generación de ideas que su implementación. Esto puede deberse a que los seguidores de líderes carismáticos se adhieren a la visión del superior en lugar de generar sus propias ideas. Este hecho puede restringir la autonomía de los empleados, que es crucial para ser creativo (Mumford, Scott, Gaddis y Strange, 2002). Por otro lado, en un estudio más reciente se encontró una alta relación entre el liderazgo transformacional ejercido por los supervisores y la conducta de innovación (no así con la creatividad) desarrollada por los subordinados con un nivel bajo de autoestima (Rank, Nelson y Xu, 2004).

Los estudios sobre la eficacia del liderazgo han intentado identificar los componentes del liderazgo eficaz y medir sus resultados. Así, Howell y Avolio (1993) encontraron que los componentes de la eficacia en el liderazgo de la alta dirección están estrechamente asociados con su apoyo a la innovación. Kisfalvi y Pitcher (2003) proporcionaron evidencias sobre el 
impacto que el carácter del director general -y por extensión, su estilo de liderazgo- tiene sobre los procesos de la organización y las decisiones estratégicas que afectan a la innovación.

La teoría sobre el liderazgo de rango total de Avolio (1999) y Bass (1985 y 1998) ha sido una de las contribuciones recientes más importante en el estudio del liderazgo (Elenkov y Manev, 2005). Básicamente se pueden distinguir cuatro componentes o factores de liderazgo transformacional, dos de liderazgo transaccional y un último factor, denominado laissez faire, que denota la ausencia de liderazgo.

Un líder es transformacional cuando, motivando a los miembros de su equipo, consigue que éstos estén dispuestos a hacer más de lo que en un principio esperaban. El primero y más importante de los factores transformacionales es el carisma, al que en los últimos años se ha denominando influencia idealizada (p.ej. Avolio y Bass, 1991; Bass, 1999; Bass y Avolio, 1994). Este factor hace referencia a la capacidad del líder para evocar una visión y ganar la confianza de sus seguidores. El carisma tiene un intenso componente emocional y crea un fuerte vínculo líder-seguidor. El segundo factor transformacional es la inspiración (o motivación inspiracional), que se define como la capacidad del líder para comunicar su visión. Un líder es inspiracional cuando estimula en sus seguidores el entusiasmo por el trabajo e incrementa la confianza en su capacidad para cumplir con éxito la misión encomendada. En este sentido, cómo se construye la confianza entre el líder y el colaborador y cómo el primero estimula la creencia en la visión del segundo, son dos aspectos centrales. El tercer factor transformacional es la estimulación intelectual o capacidad del líder para hacer que sus subordinados piensen de manera creativa e innovadora. Este tipo de líderes fomenta y estimula en sus seguidores el razonamiento, la imaginación y la resolución de problemas. Finalmente, el cuarto factor recibe el nombre de consideración individualizada y señala la capacidad del líder para prestar atención personal a todos los miembros de su equipo, haciéndoles ver que su contribución individual es importante. Los líderes que ejercen este estilo de liderazgo suelen desarrollar conductas que fomentan el crecimiento de sus seguidores, son capaces de delegar y dan información a sus subordinados.

Por su parte, el liderazgo transaccional está compuesto por los factores recompensa contingente y dirección por excepción. El primero hace referencia a las conductas del líder, mediante las que reconoce a sus subordinados por una labor bien hecha, especificando claramente la recompensa que recibirán por cada tarea. El factor dirección por excepción se refiere a aquellos líderes que intervienen solamente cuando las cosas van mal para reprender o castigar a sus subordinados. A partir del estudio de Hater y Bass (1988), las versiones del Multifactorial Leadership Questionnaire (MLQ) de Bass y Avolio (1990) distinguen dos formas de dirección por excepción: activa (el líder interviene antes de que ocurra un problema) y pasiva (el líder interviene cuando ya ha ocurrido el problema).

Por último, el factor laissez faire representa la ausencia del liderazgo o la evitación de intervenir por parte del líder.

Respecto a la relación entre el estilo transaccional y la innovación, en su estudio sobre innovación organizacional y liderazgo, Elenkov y Manev (2005) hallaron una relación significativa entre el factor recompensa contingente y el número de innovaciones organizacionales, no encontrando evidencias de relación entre la innovación y los otros dos factores. A nivel individual, es posible que la dirección por excepción inhiba la innovación y la recompensa contingente la estimule. El motivo es que mientras la primera dimensión favorece comportamientos dirigidos a evitar cometer errores, la segunda puede incentivarlos. En su estudio, Elenkov y Manev (2005) encontraron una correlación significativa entre tres de los cuatro factores del liderazgo transformacional (influencia idealizada, inspiración y consideración individualizada) y la innovación organizacional. La relación entre el clima de innovación y los estilos de liderazgo ha sido estudiada en diferentes investigaciones. Estudios recientes han relacionado el liderazgo transformacional con una visión creativa del contexto laboral (Moss, Dowling y Callanan, 2009). Este tipo de líderes desarrollan en sus colaboradores una propensión a utilizar los recursos disponibles de manera creativa. En consecuencia, los empleados dirigidos por líderes transformacionales pueden percibir un clima laboral más abierto a la innovación (Charbonnier-Voirin et al., 2010). Por otro lado, Jung, Chow y Wu (2003) encontraron evidencias de que un clima propicio para la innovación mejora la relación entre el liderazgo transformacional y la innovación organizativa. Del mismo modo, Howell y Avolio (1993) concluyeron que el clima de innovación influye positivamente en la relación entre dos dimensiones de liderazgo transformacional (estimulación intelectual y consideración individualizada) y el rendimiento del equipo. Por último, Eisenbeiss et al. (2008) relacionaron positivamente la innovación a nivel grupal con un clima de innovación y un estilo transformacional de liderazgo.

\section{Prácticas de gestión de Recursos Humanos}

Existen numerosos trabajos que indican que las prácticas de recursos humanos adoptadas por las empresas están relacionadas con la consecución de sus objetivos (p.ej., Boselie, Paauwe y Jansen, 2000; Delery y Doty, 1996; West, 1997) y su capacidad para innovar (Song, Yaping y Cass 2011). La literatura en torno al impacto de las prácticas de RR.HH. sobre los resultados organizacionales muestra tres perspectivas 
teóricas diferentes: la perspectiva universalista o de mejores prácticas, las aproximaciones de contingencia y el mejor ajuste o visión configurativa (Sánchez, 2003).

La primera, apoyada en la teoría del capital humano y la de recursos y capacidades, se refiere a la existencia de prácticas de RR.HH. cuyo efecto sobre los resultados es siempre positivo, independientemente de otros elementos internos o externos a la organización (Delaney, Lewin y Ichniowski, 1989; Huselid, 1993; 1995; Osterman, 1994; Pfeffer, 1994; Terpstra y Rozell, 1994). En segundo lugar, la perspectiva contingente sugiere que la estrategia de la organización aumenta o disminuye el impacto sobre los resultados de determinadas prácticas de RR.HH. (Butler, Ferris y Napier, 1991; Dyer, 1985; Fombrun, Tichy y Devanna, 1994; Golden y Ramanujan, 1985). Por último, la perspectiva configurativa propone que ciertas variables organizativas, entre ellas las prácticas de RR.HH., se suelen aplicar de manera conjunta, lo que da lugar a configuraciones cuya acción coordinada es la que tiene impacto sobre los resultados (Doty y Glick, 1994).

Situándonos en la perspectiva universalista, aún encontrando apoyo a las hipótesis planteadas, las prácticas a las que se refieren los diferentes autores son distintas, no existiendo unanimidad sobre qué actuaciones se pueden englobar en esta denominación genérica (Becker y Gerhart, 1996). Desde esta posición, se considera que existe una serie de prácticas de RR.HH. llamadas de alto rendimiento, alto compromiso o alta implicación (Moriones, Merino y Cerio, 2002) que facilitan la efectividad organizacional. Este tipo de prácticas están encaminadas a mejorar las capacidades del trabajador y a incrementar su motivación. Las organizaciones con prácticas de RR.HH. de alto compromiso tienden a preferir la orientación a los resultados en la gestión de sus empleados. Los niveles de calidad y rendimiento alcanzados son entendidos como consecuencia de las habilidades y conocimientos de sus miembros, más que como el producto de un alto nivel de supervisión (Bassett-Jones, 2005).

Los estudios más relevantes en esta orientación son los de Walton (1985) y Pfeffer (1994). El primero sostiene que los resultados organizativos requieren la implicación de los empleados, señalando la necesidad de un conjunto consistente de prácticas de gestión de RR.HH. para lograr ese compromiso. Frente a estas medidas, las prácticas más tradicionales persiguen el control y la adecuación del comportamiento del trabajador a los estándares definidos previamente. A diferencia de la gestión de RR.HH. orientada al compromiso, la gestión de RR.HH. orientada al control se preocupa principalmente por la reducción de costes laborales y busca la eficiencia a través del cumplimiento de las reglas y los procedimientos (Arthur, 1994).

La gestión de RR.HH. orientada al compromiso debería desarrollar en los empleados las actitudes deseadas, a través del establecimiento de conexiones psicológicas entre sus objetivos y los de la organización. Se piensa, además, que en las organizaciones con este tipo de gestión será más probable encontrar personas con una conducta organizativa favorable (Organ, 1988), ofreciendo un desempeño más allá de las especificaciones de su puesto, sin esperar una recompensa por ello, mostrando así comportamientos que resultan críticos para el éxito de la organización (Arthur, 1994). Por oposición a lo que sucede con el rendimiento establecido formalmente en el trabajo, conductas como ayudar a otras personas, mostrar iniciativa, o defender la reputación de la empresa frente a los demás, no se desarrollan si no se presenta una ocasión adecuada para ello. Es por eso que Van Kinppenberg y Ellemers (2004) señalan que tales conductas resultan difíciles de predecir y más difíciles aún de motivar. El rendimiento formal puede motivarse a través de sistemas de objetivos e incentivos. Sin embargo, estos procedimientos estimulan motivaciones centradas en el interés personal y pueden inhibir la proactividad de los empleados.

Según Whitener (2001), los empleados interpretan las acciones y prácticas de gestión de RR.HH. como señales del compromiso de la organización. En reciprocidad, los empleados corresponden mejorando la percepción sobre su propio compromiso con la organización. Dorenbosch, van Engen y Verhagen (2005) hallaron evidencias de una relación positiva entre las prácticas de RR.HH. orientadas al compromiso y el sentimiento de propiedad del trabajo. Esto avala la idea de que el comportamiento del empleado es, efectivamente, recíproco a su percepción sobre el comportamiento de la organización con él. Inspirados en este último estudio, en este trabajo consideramos que las prácticas de RR.HH. estarán relacionadas con el clima de innovación, facilitando o inhibiendo su percepción.

La investigación relativa al impacto de las prácticas de RR.HH. sobre el clima y la cultura organizacional ha sido escasa (Rogg, Schmidt, Shull y Schmitt, 2001). Algunos estudios han relacionado, por ejemplo, prácticas de downsizing con una percepción general negativa del clima organizacional (Luthans y Sommer, 1999). Tesluk, Farr, Mathieu y Vance (1995) relacionaron una mayor cantidad de formación recibida por los empleados con un mayor grado de su compromiso con la organización. Esta relación entre prácticas de RR.HH. y clima organizacional es coherente con el modelo de Atracción-Selección-Abandono (Schneider, 1987). De esta forma, las personas que permanecen en una organización tienden a compartir percepciones acerca de ella y su entorno. Así, las prácticas de RR.HH. pueden fomentar un clima organizacional positivo en relación con los objetivos de la organización. Por ejemplo, la formación, la información compartida y la retribución pueden transmitir la importancia de las metas organizacionales. 


\section{Justificación de la investigación y objeto de estudio}

El marcado carácter social de la innovación implica que factores como el clima organizacional resulten especialmente relevantes. Las variables analizadas en este estudio tienen que ver con el grado en que el entorno organizacional favorece la innovación, de acuerdo con la teoría Atracción-Selección-Abandono de Schneider (1987). Inicialmente a través del líder, que proporciona interpretaciones que el grupo tiende a aceptar, y que pueden influir en su percepción del clima organizacional. Y posteriormente a través de las políticas y prácticas de la organización, dirigidas a la consecución de sus fines (Schneider, Goldstein y Smith, 1995).

Partiendo del modelo de Schneider, el objetivo de este estudio es identificar aquellos estilos de liderazgo y prácticas de gestión de RR.HH. que favorecen la percepción de un clima organizacional de innovación. En el marco de este objetivo, nuestro estudio plantea las siguientes hipótesis:

En relación a los estilos de liderazgo, se espera que las personas dirigidas por líderes transformacionales desarrollen una mayor percepción del clima de innovación. Especialmente en la dimensión de inspiración, en la que el líder es capaz de motivar a sus colaboradores a través de la evocación de la visión organizacional. Este estímulo incrementa en el empleado la confianza en su capacidad para desarrollar la misión, influyendo también en la percepción de su entorno.

Hipótesis 1: La dimensión de liderazgo inspiracional estará más relacionada con la percepción un clima innovador y ofrecerá una mayor capacidad predictiva, frente al resto de dimensiones de los tres estilos de liderazgo.

Respecto a las prácticas de gestión de RR.HH., esperamos que las relacionadas con el estímulo de la participación de los empleados estén más relacionadas con el clima de innovación. Frente al resto de dimensiones, el éxito de las prácticas dirigidas a estimular la participación se basa en que el empleado perciba que su entorno le permite intervenir de manera activa.

Hipótesis 2: Las prácticas dirigidas a estimular la participación estarán más relacionadas con la percepción un clima innovador y ofrecerán una mayor capacidad predictiva, frente al resto de prácticas de gestión de RR.HH.

Por último, se espera que al considerar las variables de liderazgo y gestión de RR.HH. conjuntamente, ambos tipos de variables mantengan su capacidad predictiva sobre el clima de innovación.

Hipótesis 3: Al incluir en el mismo modelo de regresión las dimensiones de liderazgo y prácticas de ges- tión significativas en los modelos de regresión previos, ambos tipos de variables mantendrán su capacidad predictiva.

\section{Método}

\section{Muestra}

La muestra está compuesta por 458 empleados pertenecientes a 16 empresas, mayoritariamente privadas. Estas organizaciones operan en los sectores de transporte, producción industrial, energía, consultoría, tecnología, sanidad, educación, distribución de alimentación y distribución de suministros industriales. Excepto la organización sanitaria, que corresponde a un hospital público, el resto de organizaciones son empresas privadas. 13 empresas son PYMES y tres son empresas de carácter multinacional con más de 250 empleados. Respecto a la composición de la muestra, $218(47.6 \%)$ son hombres y $211(46.1 \%)$ son mujeres. La media de edad es de 36.9 años y el $50 \%$ se concentra entre 29 y 44 años. La antigüedad media en la empresa es de 10.8 años y el 50\% tiene una antigüedad comprendida entre los 2 y los 18 años. Respecto al puesto ocupado, el $14.8 \%$ son operarios, el $21.2 \%$ administrativos, el $41.7 \%$ técnicos, el $15.1 \%$ se engloban en la categoría directores, gerentes, jefes o supervisores y el $3.3 \%$ se ubican en la categoría otros puestos, diferentes a los propuestos. El $72.5 \%$ tienen una relación laboral de carácter indefinido, mientras que el $18.3 \%$ una relación laboral de tipo temporal. De manera minoritaria, el $4.6 \%$ son cooperativistas. Finalmente, respecto al nivel de estudios, más de la mitad de los sujetos tienen un nivel universitario (57.9\%), mientras que el $26.0 \%$ tiene estudios medios equivalentes a Formación Profesional o Bachillerato, el $10.3 \%$ tiene estudios básicos equivalentes a Educación General Básica o similar y tan solo el $2.0 \%$ no tiene ningún tipo de formación reglada.

\section{Medidas}

Clima de innovación. El clima organizacional para la innovación se ha medido a través de una de las escalas del Organizational Climate Measure (OCM) de Patterson et al. (2005), concretamente la escala referida a la flexibilidad y la innovación. Basado en el modelo de los valores en competencia de Quinn y Rohrbaugh (1983), el OCM consta de 17 escalas y propone cuatro cuadrantes principales del clima organizacional con diversas subescalas.

La escala de Flexibilidad e Innovación está incluida en el modelo de sistemas abiertos y originalmente se nutre de dos escalas independientes. La dimensión de flexibilidad, como una orientación hacia el cambio, se ha utilizado en diversos estudios (p.ej., Garrahan y 
Stewart, 1992; King y Anderson, 1995). Por otro lado, la dimensión de innovación, como el grado de estímulo y apoyo a nuevas ideas e innovaciones ha sido utilizada por West y Farr (1990).

De acuerdo con la propuesta original, el cuestionario plantea una serie de afirmaciones sobre la organización, de manera que el sujeto debe responder según le parezca aplicable a la empresa cada enunciado. La escala propone cuatro alternativas de respuesta, siendo 1; Totalmente falso, 2; Bastante falso, 3; Bastante cierto, 4; Totalmente cierto. La interpretación de los resultados se realiza de forma que una puntuación mayor indica una mayor presencia percibida del clima para la innovación. La escala de Flexibilidad e Innovación consta de seis ítems y presenta una fiabilidad de $\alpha .91$.

Estilos de liderazgo. En esta investigación se utiliza la versión actual del Multifactorial Leadership Questionnaire (MLQ), en la forma 5x. Contiene 45 ítems, de los cuáles 36 representan los nueve factores del liderazgo mencionados anteriormente (cuatro ítems por cada factor de liderazgo) y 9 ítems que evalúan tres escalas de resultados del liderazgo. En concreto utilizaremos los 36 ítems que corresponden a los nueve factores de liderazgo: las dimensiones transformacionales estimulación intelectual, inspiración, consideración individualizada y la de influencia idealizada (también denominada carisma), que distingue una dimensión conductual y otra atribuida; las dimensiones transaccionales dirección por excepción activa, dirección por excepción pasiva y recompensa contingente; y la dimensión de no liderazgo o laissez-faire.

El cuestionario plantea cinco alternativas de respuesta: 1; Nunca, 2; Rara Vez, 3; A Veces, 4; A Menudo, 5; Frecuentemente, sino siempre. La interpretación de los resultados se realiza de manera que una puntuación mayor en cada escala indica una mayor presencia percibida del estilo de liderazgo correspondiente. Los índices de fiabilidad de cada una de las escalas (ver tabla 1) ofrecen valores satisfactorios, salvo la dimensión dirección por excepción pasiva, razón por la que esta dimensión no se ha incorporado en los análisis realizados.

Prácticas de gestión de Recursos Humanos. La escala utilizada fue desarrollada por Boselie, Hesselink, Paauwe y van der Wiele (2001). A través de esta escala, los empleados pueden indicar el grado en que, según su percepción, las distintas prácticas están implementadas y extendidas en su empresa. Consta de cinco alternativas de respuesta que exploran el grado de acuerdo con las diferentes afirmaciones que se realizan en el cuestionario (1; Muy en desacuerdo, 2; En desacuerdo, 3; Ni de acuerdo ni en desacuerdo, 4; De acuerdo, 5; Muy de acuerdo).

Una puntuación baja señala una percepción de baja presencia de las prácticas de gestión orientadas al compromiso; por el contrario, una puntuación alta indica una percepción de que las prácticas de gestión orientadas al compromiso son frecuentes en la empresa. La fiabilidad de las escalas (alfa de Cronbach) ofrece valores satisfactorios en las cuatro dimensiones (ver Tabla 1).

\section{Resultados}

El contraste de las hipótesis se ha realizado a través del análisis de correlaciones entre las variables y diferentes modelos de regresión jerárquica. Es posible que, en la medida en que el clima de apoyo a la innovación se valora a priori como positivo y dependa de las prácticas de la dirección, los niveles altos de la organización perciban que existe un apoyo mayor que el percibido por los niveles inferiores. Por este motivo, en los análisis de regresión previos se ha introducido como variable de control el puesto ocupado. Tras el análisis de correlaciones, se han realizado diferentes análisis de regresión considerando como variables independientes las dimensiones de liderazgo. Estas dimensiones se han introducido en el modelo de regresión, agrupadas en tres bloques, según su pertenencia al estilo transfor-

Tabla 1. Estadísticos descriptivos y coeficientes de correlación entre las variables contempladas en el estudio

\begin{tabular}{lccccccccccccccccc}
\hline Variables & Media & Sd & alfa & 1 & 2 & 3 & 4 & 5 & 6 & 7 & 8 & 9 & 10 & 11 & 12 \\
\hline 1. Clima de innovación & 2.52 & 0.663 & .91 & & & & & & & & & & \\
2. Influencia idealizada atribuida & 3.32 & 0.917 & .80 & $.477^{* *}$ & & & & & & & & \\
3. Influencia idealizada conductual & 3.28 & 0.823 & .73 & $.490^{* *}$ & $.716^{* *}$ & & & & & & & \\
4. Inspiración (Motiv. Inspiracional) & 3.48 & 0.941 & .89 & $.535^{* *}$ & $.758^{* *}$ & $.757^{* *}$ & & & & & & & \\
5. Estimulación intelectual & 3.24 & 0.774 & .78 & $.465^{* *}$ & $.730^{* *}$ & $.686^{* *}$ & $.719^{* *}$ & & & & & \\
6. Consideración individualizada & 3.07 & 0.850 & .70 & $.499^{* *}$ & $.788^{* *}$ & $.677^{* *}$ & $.690^{* *}$ & $.714^{* *}$ & & & & \\
7. Recompensa contingente & 3.18 & 0.862 & .78 & $.518^{* *}$ & $.757^{* *}$ & $.716^{* *}$ & $.728^{* *}$ & $.717^{* *}$ & $.772^{* *}$ & & & \\
8. Dirección por excepción activa & 3.05 & 0.738 & .66 & $.136^{* *}$ & $.318^{* *}$ & $.331^{* *}$ & $.220^{* *}$ & $.333^{* *}$ & $.214^{* *}$ & $.303^{* *}$ & & \\
9. Laissez faire & 2.21 & 0.795 & .70 & $-.231^{* *}$ & $-.482^{* *}$ & $-.349^{* *}$ & $-.411^{* *}$ & $-.330^{* *}$ & $-.420^{* *}$ & $-.441^{* *}$ & $-.098^{*}$ & & \\
10. Información compartida & 3.28 & 0.992 & .91 & $.565^{* *}$ & $.366^{* *}$ & $.458^{* *}$ & $.467^{* *}$ & $.392^{* *}$ & $.390^{* *}$ & $.377^{* *}$ & .057 & $-.167^{* *}$ & \\
11. Participación del empleado & 3.05 & 1.026 & .89 & $.738^{* *}$ & $.480^{* *}$ & $.549^{* *}$ & $.563^{* *}$ & $.484^{* *}$ & $.540^{* *}$ & $.532^{* *}$ & .087 & $-.207^{* *}$ & $.688^{* *}$ & \\
12. Formación y desarrollo & 3.26 & 0.919 & .84 & $.524^{* *}$ & $.416^{* *}$ & $.417^{* *}$ & $.437^{* *}$ & $.384^{* *}$ & $.419^{* *}$ & $.500^{* *}$ & $.153^{* *}-.226^{* *}$ & $.461^{* *}$ & $.574^{* *}$ \\
13. Sistema retributivo & 2.63 & 0.890 & .80 & $.425^{* *}$ & $.276^{* *}$ & $.282^{* *}$ & $.245^{* *}$ & $.295^{* *}$ & $.312^{* *}$ & $.346^{* *}$ & $.134^{* *}-.058$ & $.461^{* *}$ & $.514^{* *} .328^{* *}$
\end{tabular}

$* p<0.05 ; * * p<0.01$ 
Tabla 2. Modelo de regresión del clima de innovación con estilos de liderazgo

\begin{tabular}{|c|c|c|c|c|c|c|}
\hline & Modelo & Beta & $\mathrm{t}$ & Signif. & $\mathrm{R}^{2}$ corregida & $\Delta \mathrm{R}^{2}(\mathrm{p})$ \\
\hline \multirow[t]{2}{*}{1} & (Constante) & & 25.92 & 0.000 & & \\
\hline & Puesto ocupado & 0.08 & 1.624 & 0.105 & 0.004 & $0.04(.107)$ \\
\hline \multirow[t]{7}{*}{2} & (Constante) & & 6.661 & 0.000 & & \\
\hline & Puesto ocupado & 0.006 & 0.140 & 0.888 & & \\
\hline & Influencia idealizada (atribuida) & -0.037 & -0.47 & 0.641 & & \\
\hline & Influencia idealizada (conductual) & 0.135 & 1.969 & 0.05 & & \\
\hline & Inspiración (Motiv. inspiracional) & 0.267 & 3.638 & 0.000 & & \\
\hline & Estimulación intelectual & 0.051 & 0.757 & 0.45 & & \\
\hline & Consideración individualizada & 0.221 & 3.101 & 0.002 & 0.317 & $0.313(.000)$ \\
\hline \multirow[t]{9}{*}{3} & (Constante) & & 5.818 & 0.000 & & \\
\hline & Puesto ocupado & 0.016 & 0.389 & 0.697 & & \\
\hline & Influencia idealizada (atribuida) & -0.055 & -0.690 & 0.491 & & \\
\hline & Influencia idealizada (conductual) & 0.115 & 1.627 & 0.104 & & \\
\hline & Inspiración (Motiv. inspiracional) & 0.231 & 3.073 & 0.002 & & \\
\hline & Estimulación intelectua & 10.033 & 0.481 & 0.631 & & \\
\hline & Consideración individualizada & 0.16 & 2.111 & 0.035 & & \\
\hline & Recompensa contingente & 0.171 & 2.273 & 0.024 & & \\
\hline & Dirección por excepción activa & -0.027 & -0.600 & 0.552 & 0.322 & $0.009(.071)$ \\
\hline \multirow[t]{10}{*}{4} & (Constante) & & 3.631 & 0.000 & & \\
\hline & Puesto ocupado & 0.016 & 0.382 & 0.702 & & \\
\hline & Influencia idealizada (atribuida) & -0.036 & -0.44 & 0.663 & & \\
\hline & Influencia idealizada (conductual) & 0.113 & 1.603 & 0.11 & & \\
\hline & Inspiración (Motiv. inspiracional) & 0.235 & 3.120 & 0.002 & & \\
\hline & Estimulación intelectual & 0.025 & 0.361 & 0.719 & & \\
\hline & Consideración individualizada & 0.163 & 2.157 & 0.032 & & \\
\hline & Recompensa contingente & 0.182 & 2.402 & 0.017 & & \\
\hline & Dirección por excepción activa & -0.029 & -0.630 & 0.527 & & \\
\hline & Laissez faire & 0.053 & 1.120 & 0.263 & 0.322 & $0.000(.263)$ \\
\hline
\end{tabular}

macional, transaccional o laissez faire. A continuación se han repetido los análisis de regresión sobre la variable dependiente considerando las prácticas de gestión. Finalmente, los modelos de regresión se han realizado considerando aquellas variables que se han mostrado predictoras significativas en los análisis previos.

La tabla 1 muestra, junto con los resultados descriptivos de las variables del estudio, los resultados de los análisis de correlaciones en los que se aprecia un número elevado de correlaciones significativas. Respecto a las dimensiones de liderazgo, se observa que las cinco dimensiones transformacionales muestran relaciones positivas con el clima organizacional, en torno a .50, siendo la más alta la de inspiración $(\mathrm{r}=.535, p<.01)$, tal como se plantea en las hipótesis. La dimensión de recompensa contingente también muestra una relación positiva $(\mathrm{r}=.518, p<.01)$, siendo mucho menor la de dirección por excepción $(\mathrm{r}=.136, p<.01)$. La dimensión de laissez-faire muestra una relación negativa con el clima innovador $(\mathrm{r}=-.231, p<.01)$.

En cuanto a las correlaciones entre el clima organizacional y las prácticas de RRHH orientadas al compromiso, la más elevada corresponde a la participación de los empleados, tal como se esperaba $(r=.738, p<$ .01 ), seguida de la información compartida y la forma- ción y desarrollo, por encima de .50 , siendo la menos elevada la de retribución $(\mathrm{r}=.431, p<.01)$. Estas diferencias sugieren que la percepción de un clima innovador parece estar más relacionada con prácticas orientadas a estimular una relación más intrínseca entre la organización y sus miembros.

El análisis de la capacidad predictiva de los estilos de liderazgo sobre el clima de innovación se ha realizado a través de un análisis de regresión considerando los tres estilos de liderazgo identificados por Bass y Avolio. Como variable de control se ha introducido el puesto ocupado. El modelo de regresión explica el $32.2 \%$ de la varianza del clima innovador.

El análisis de los datos de la tabla 2 muestra que únicamente las dimensiones inspiración, consideración individualizada y recompensa contingente ofrecen valores significativos. El análisis del peso relativo de cada dimensión confirma la hipótesis 1 y sugiere que la capacidad del líder de comunicar su visión o motivación inspiracional ofrece un valor superior $(\beta=$ .235) y una correlación más alta con el clima de innovación $(\mathrm{r}=.535, p<.01)$ que el resto de dimensiones de liderazgo. A continuación, se sitúa la recompensa del líder a sus colaboradores por la labor bien hecha o recompensa contingente $(\beta=.182)$ y la capacidad del 
Tabla 3. Modelo de regresión del clima de innovación con prácticas de gestión de RR.HH

\begin{tabular}{|c|c|c|c|c|c|c|}
\hline & Modelo & Beta & $\mathrm{t}$ & Signif. & $\mathrm{R}^{2}$ corregida & $\Delta \mathrm{R}^{2}(\mathrm{p})$ \\
\hline \multirow[t]{2}{*}{1} & (Constante) & & 26.300 & 0.000 & & \\
\hline & Puesto ocupado & 0.093 & 1.940 & 0.053 & 0.006 & $0.006(.053)$ \\
\hline \multirow[t]{3}{*}{2} & (Constante) & & 13.340 & 0.000 & & \\
\hline & Puesto ocupado & -0.061 & -1.460 & 0.145 & & \\
\hline & Información compartida & 0.573 & 13.750 & 0.000 & 0.310 & $0.304(.000)$ \\
\hline \multirow[t]{4}{*}{3} & (Constante) & & 12.260 & 0.000 & & \\
\hline & Puesto ocupado & -0.059 & -1.760 & 0.080 & & \\
\hline & Información compartida & 0.113 & 2.495 & 0.013 & & \\
\hline & Participación del empleado & 0.673 & 15.170 & 0.000 & 0.551 & $0.241(.000)$ \\
\hline \multirow[t]{5}{*}{4} & (Constante) & & 9.272 & 0.000 & & \\
\hline & Puesto ocupado & -0.055 & -1.640 & 0.101 & & \\
\hline & Información compartida & 0.096 & 2.137 & 0.033 & & \\
\hline & Participación del empleado & 0.604 & 12.650 & 0.000 & & \\
\hline & Formación y desarrollo & 0.141 & 3.616 & 0.000 & 0.564 & $0.014(.000)$ \\
\hline \multirow[t]{6}{*}{5} & (Constante) & & 8.614 & 0.000 & & \\
\hline & Puesto ocupado & -0.053 & -1.590 & 0.112 & & \\
\hline & Información compartida & 0.091 & 1.987 & 0.048 & & \\
\hline & Participación del empleado & 0.596 & 12.060 & 0.000 & & \\
\hline & Formación y desarrollo & 0.14 & 3.589 & 0.000 & & \\
\hline & Sistema retributivo & 0.023 & 0.608 & 0.543 & 0.563 & $-0.001(.543)$ \\
\hline
\end{tabular}

líder para prestar atención personal a sus colaboradores, haciéndoles ver que su contribución individual es importante o consideración individualizada $(\beta=.163)$.

Estos datos son congruentes con los obtenidos por Elenkov y Manev (2005), que encontraron las relaciones más importantes entre liderazgo y conductas de innovación con las dimensiones de inspiración motivacional entre las dimensiones transformacionales y la recompensa contingente entre las transaccionales.

En el siguiente análisis se ha considerado la capacidad predictiva de las prácticas de gestión de Recursos Humanos sobre la variable criterio. Como en el apartado anterior, se ha introducido el puesto ocupado como variable de control. Posteriormente cada práctica se ha introducido en pasos sucesivos del análisis de regre- sión. El modelo obtenido explica el $56.3 \%$ de la varianza de la variable criterio.

Los datos de la tabla 3 muestran que, salvo el sistema retributivo, las prácticas de información compartida, participación y formación ofrecen valores significativos. El análisis pormenorizado de los coeficientes beta sugiere que, de acuerdo con lo esperado en la hipótesis 2, por encima del resto de prácticas las dirigidas a estimular la participación son un potente predictor del clima de innovación $(\beta=.596)$ y ofrecen la correlación más alta del estudio $(\mathrm{r}=.738, p<.01)$. En menor medida, también predicen significativamente el clima de innovación las prácticas dirigidas a la formación y el desarrollo de los empleados $(\beta=.140)$ y las prácticas dirigidas a compartir información relevante de la organización $(\beta=.091)$.

Tabla 4. Modelo de regresión final del clima de innovación

\begin{tabular}{|c|c|c|c|c|c|c|}
\hline & Modelo & Beta & $\mathrm{t}$ & Signif. & $\mathrm{R}^{2}$ corregida & $\Delta \mathrm{R}^{2}(\mathrm{p})$ \\
\hline \multirow[t]{4}{*}{1} & (Constante) & & 8.828 & 0.000 & & \\
\hline & Inspiración (Motiv. inspiracional) & 0.297 & 4.887 & 0.000 & & \\
\hline & Consideración individualizada & 0.161 & 2.478 & 0.014 & & \\
\hline & Recompensa contingente & 0.177 & 2.569 & 0.011 & 0.327 & $0.327(.000)$ \\
\hline \multirow[t]{7}{*}{2} & (Constante) 6.3660 .000 & & & & & \\
\hline & Inspiración (Motiv. inspiracional) & 0.086 & 1.712 & 0.088 & & \\
\hline & Consideración individualizada & 0.034 & 0.658 & 0.511 & & \\
\hline & Recompensa contingente & 0.071 & 1.263 & 0.207 & & \\
\hline & Información compartida & 0.075 & 1.713 & 0.087 & & \\
\hline & Participación del empleado & 0.527 & 10.47 & 0.000 & & \\
\hline & Formación y desarrollo & 0.098 & 2.444 & 0.015 & 0.578 & $0.251(.000)$ \\
\hline
\end{tabular}


En el último bloque de análisis se ha puesto a prueba la capacidad predictiva de aquellas variables que han mostrado ser significativas en los análisis previos. El objetivo es identificar de entre todas las variables de liderazgo y de prácticas de gestión de RR.HH. aquéllas que poseen la mayor capacidad de incidir en la percepción de un clima organizacional innovador. El método de introducción de las variables ha sido en dos bloques, el primero incluye las dimensiones de liderazgo y el segundo las prácticas de gestión.

El modelo obtenido explica el $57.8 \%$ de la varianza y la inclusión de las prácticas de gestión ha supuesto un incremento de .251 en el valor de $\mathrm{R}^{2}$ corregida (corrección a la baja de $\mathrm{R}^{2}$ basada en el número de casos y de variables independientes), respecto a la capacidad predictora del liderazgo $\left(\mathrm{R}^{2} \mathrm{c}=.327\right)$. El valor de los coeficientes se aprecia en la tabla 4, resultando significativas únicamente las prácticas dirigidas a estimular la participación y la formación de los empleados. Es decir, en contra de lo esperado en la hipótesis 3 , al introducir de manera conjunta las dimensiones de liderazgo y las prácticas de gestión de RR.HH., las primeras han cedido su capacidad predictiva a las segundas. El análisis de los valores beta sugiere que las prácticas dirigidas a estimular la participación $(\beta=.527)$ ofrecen un peso relativo mayor que las prácticas dirigidas a la formación y el desarrollo de los empleados $(\beta=.098)$.

\section{Discusión}

Diversos estudios sobre los antecedentes de la innovación organizacional han señalado la importancia del clima de innovación. Por ejemplo, en una comparación entre empresas innovadoras y no innovadoras, Ekvall (1996) identificó en las diferencias entre sus climas de innovación el principal factor diferencial entre ellas. Desde esta perspectiva resulta interesante conocer qué aspectos organizacionales facilitan la percepción del clima de innovación por parte de los empleados. Este estudio analiza la relación entre los estilos de liderazgo y las prácticas de RR.HH. con la percepción del clima organizacional de innovación. Cuando el individuo percibe un clima laboral de apoyo a la producción de innovaciones, un estilo de dirección que le estimula y unas prácticas de gestión que le animan a innovar, es lógico pensar que dedicará más recursos a la generación, promoción y realización de innovaciones, de manera que éstas resultan más efectivas. Esto es, consistentemente con Nicholson y West (1988), la percepción de trabajar en una organización o un equipo, que apoya la innovación, genera en el individuo la confianza necesaria para centrar sus esfuerzos en incrementar la efectividad de sus innovaciones.

Los estilos de liderazgo y las prácticas de gestión de RR.HH. son dos importantes vías de interacción entre la organización y sus miembros. A través de estos contactos los empleados desarrollan una percepción acer- ca de los elementos fundamentales de su organización. De acuerdo con la teoría Atracción-SelecciónAbandono de Schneider (1987), los miembros de una organización van configurando con el tiempo una visión homogénea de la misma. Un ejemplo de este tipo de percepciones es el clima para la innovación. Además, en el marco del contrato psicológico, este tipo de señales organizacionales definen las creencias del individuo en cuanto a las obligaciones recíprocas con su organización (Rousseau, 1990).

Inicialmente se ha abordado la relación entre el clima de innovación y los estilos de liderazgo. En estos análisis la percepción de un clima innovador se ha relacionado positivamente con las dimensiones transformacionales y transaccionales. Por otro lado, los análisis de regresión jerárquica señalan a la dimensión inspiración como la mejor predictora del clima innovador. El estilo inspiracional, dirigido a que los colaboradores asuman y cumplan la misión, hace que se genere la autoconfianza suficiente para defender las nuevas ideas y se consigan los apoyos necesarios para su desarrollo. Así es como un empleado dirigido de forma inspiradora puede percibir el clima de su empresa como más innovador. Es decir, generando la percepción de que su entorno laboral es más permeable a las ideas innovadoras. Estos resultados coinciden con los encontrados por Pons (2009). Este estudio analizó los antecedentes de la generación, promoción y realización de ideas innovadoras como fases de la conducta innovadora. Por encima de otras dimensiones organizacionales y estilos de liderazgo, el clima de innovación y el liderazgo inspiracional se mostraron como potentes predictores de la etapa de promoción de las innovaciones. Sin embargo, no mostraron una capacidad predictiva significativa en las otras dos fases en las que la innovación no requiere interacción social.

El liderazgo contingente también ha mostrado una capacidad significativa para predecir el clima innovador. Posiblemente esta capacidad esté relacionada con los postulados generales de las teorías del intercambio social. Muchas de estas teorías tratan de explicar los procesos de distribución e intercambio de bienes y recursos a través de un principio general de comportamiento recíproco y colectivo (Fernández-Ríos, 1999). Desde esta perspectiva, la percepción del clima como innovador podría depender de sus percepciones de equidad o inequidad. A su vez, esta percepción es el resultado del proceso de comparación social cuando se establecen relaciones interpersonales y de intercambio con la organización (Gil-Monte, 2001), especialmente a través de la relación con el superior jerárquico. Un aspecto central en estas teorías se refiere a la reciprocidad. Este concepto, difícil de definir, no significa $d o$ ut des ("Doy para que des"), sino un intercambio simbólico en el que una parte da con la expectativa de que el otro, cuándo y cómo pueda, dé lo que pueda en términos de equivalencia simbólica (Donati, 1997).

La tercera dimensión de liderazgo que se ha mostra- 
do como predictora del clima de innovación ha sido la consideración individualizada. Mediante esta dimensión, el líder transformacional desarrolla la autoconfianza del seguidor. Este fortalecimiento psicológico puede llevar a los individuos a sentirse capaces de realizar tareas más allá de las formalmente establecidas en su puesto de trabajo (Fuller, Marler y Hester, 2006). Como en la inspiración, este estilo de liderazgo puede provocar en el seguidor la percepción de que el clima organizacional es más favorable al desarrollo de tareas emergentes. En ambos casos, en línea con la teoría mantenida por Seers (1989), la percepción de apoyo por parte de los superiores, genera un clima facilitador de la innovación. Posiblemente, mediante la confianza generada para administrar sus propios recursos. A pesar de su relación con el clima de innovación, las prácticas de RR.HH. explican la varianza explicada por el liderazgo, reduciendo esa relación significativa. Sólo la dimensión de inspiración se mantiene cerca de la significación estadística $\beta .=086, p=.088$ ).

Por encima de los estilos de liderazgo, las prácticas de gestión de RR.HH. han mostrado una mayor capacidad para predecir el clima de innovación. Los cuatro tipos de prácticas consideradas han mostrado una relación positiva con la percepción de clima. Estas relaciones refuerzan la sugerencia de Whitener (2001) sobre cómo los empleados interpretan las acciones y prácticas de RR.HH. como señales del compromiso de la organización. Así, la percepción del empleado sobre las prácticas de gestión de su organización se relaciona con la percepción de un clima organizacional facilitador de la innovación y el compromiso, tal como señalaban Mowday, Steers y Porter (1979).

Destaca el papel desempeñado por las prácticas dirigidas a estimular la participación. Por encima del resto de variables, estas prácticas han mostrado una alta correlación y capacidad predictiva sobre el clima de innovación, hasta el punto que reducen drásticamente la capacidad predictora del liderazgo y del resto de prácticas de RR.HH. Desde una perspectiva universalista se asume que las prácticas de participación producen un efecto positivo sobre la organización (Walton, 1985) y sus atributos, como el clima organizacional (Fernie, Metcalf y Woodland, 1995). De esta forma, cuando el individuo percibe que su organización le estimula a participar la asocia a la posibilidad de innovar. Esta relación es importante en la medida en que la percepción de poder innovar implica la posibilidad, no solo de participar, sino de correr el riesgo de equivocarse. Esto es, para que el estímulo de la participación sea efectivo no debe limitarse al desarrollo de las tareas establecidas en el puesto, debe ampliarse a la posibilidad de participar desarrollando tareas emergentes. La participación en la toma de decisiones también se ha relacionado positivamente con el grado de compromiso con la organización (Wallace, 1995). De este modo, la asunción de los riesgos inherentes a la participación puede ser consecuencia de un mayor nivel de compromiso.
La segunda práctica que ha mostrado una relación y una capacidad significativa para predecir el clima de innovación ha sido la formación y el desarrollo de las personas. Numerosos estudios han relacionado estas prácticas con resultados organizacionales como la calidad del producto, la cuota de mercado o el crecimiento de las ventas (Kalleberg y Moody, 1994). La relación encontrada en este estudio sugiere además que también afecta a la percepción de la predisposición organizacional hacia la innovación. Esta relación es coherente con los hallazgos de otros estudios (p.ej., Delaney y Huselid, 1996; Harel y Tzafrir, 1999) en los que las prácticas de formación afectaron de manera positiva al desempeño organizacional percibido.

Finalmente, las prácticas dirigidas a compartir información relevante entre los miembros de la organización también se han mostrado como predictoras significativas del clima de innovación. El acceso a la información transmite confianza a los empleados y mejora la calidad de sus decisiones (Vlachos, 2009). Esta práctica se ha relacionado positivamente con resultados organizacionales como la productividad y la rentabilidad y negativamente con los costes laborales (Morishima, 1991). Además, el hecho de servir de feedback sobre el desempeño puede ayudar al desarrollo de la innovación como estrategia de superación de los problemas detectados (Chow, Harrison, McKinnon y Wu, 1999). Por otro lado, el acceso a información relevante permite la promoción profesional, generalmente desarrollando nuevas funciones (Burguess, 2005). Así, parece que a través del proceso de reciprocidad descrito anteriormente, informar de manera proactiva sobre la estrategia y los resultados de la organización contribuye a que el individuo perciba un clima más favorable hacia el cambio y la innovación. No obstante, la capacidad predictora de esta variable se ve reducida por la de las dos dimensiones de prácticas de RR.HH. anteriores $(\beta=.075, p=.087)$.

En conclusión, los resultados obtenidos refuerzan la relación existente entre el estilo de liderazgo desarrollado y la percepción de un clima organizacional innovador. Y por encima de esta relación, que el clima es percibido como innovador cuando los miembros de la organización perciben que ésta les permite participar activamente y les capacita para desarrollar nuevas competencias. A pesar de las limitaciones del estudio (estudio correlacional; muestra perteneciente a empresas de distintos sectores y tipos de actividad, lo que puede suponer diferentes demandas de conducta innovadora; posibilidad de sesgos en los resultados por la varianza de método común de los instrumentos de medida utilizados), los resultados obtenidos sugieren que las empresas que deseen fomentar un clima innovador deberían fomentar prácticas de RR.HH. que estimulen la participación de los empleados, su formación y desarrollo y deberían promover estilos de liderazgo que desarrollen la inspiración de los trabajadores. 


\section{Extended Summary}

\section{Introduction}

Innovation became one of the main competitive advantages of developed economies (Chesnais, 1990), increasing the interest for innovation research (Anderson, De Dreu and Nijstad, 2004; Ford and Gioia, 1995; King and Anderson, 2002; Van de Ven, Angle and Poole, 1989; West, 2002). Development of new ideas requires certain level of social interaction before they are considered as innovations, giving importance to contextual factors like organizational climate. Innovation climate (Anderson and West, 1992; Eisenbeiss, van Knippenberg and Boerner, 2008; Scott and Bruce, 1994) stimulates employees to contribute in a creative manner to organizations.

Recent studies related innovation climate with leadership styles and Human Resources Management (HRM) (e.g. Charbonnier-Voirin, El Akremi, and Vandenberghe, 2010; Chang, Gong and Shum, 2011). Employee perceptions of supervisor behavior and company treatment through practices and policies are relevant aspects that influence their motivation and climate perceptions. Baer and Frese (2003) outlined the need to develop climates that make feel employees secure when assuming risks, proposing ideas or speaking about problems.

Creative and innovative work is ambiguous, risky and subject to criticism, so supervisory support will facilitate creativity, while control-oriented supervision will inhibit it (Amabile and Gryskiewicz, 1989; West and Farr, 1989). Janssen (2005) found that perceptions of supervisory support increased workers capacity to influence creatively on their work environment, as supervisors give or neglect support for promoting the development and use of new ideas. Leadership showed significant relationship with implementation of innovative ideas (Axtell, Holman, Unsworth, Wall, Waterson, and Harrington, 2000), and supervisors' interpretation and responses to employees' innovative ideas (Janssen, De Vliert and West, 2004), among other variables.

Transformational and transactional leadership styles were related with innovation (Elenkov and Manev, 2005) and innovative climate. Transformational leadership was linked with a creative vision of work environment (Moss, Dowling, and Callanan, 2009), employees with transformational leaders perceived their organizational climate as more open to innovations (Charbonnier-Voirin et al., 2010), and innovative climate improved the relationship between transformational leadership and organizational innovations (Jung, Chow, and $\mathrm{Wu}, 2003$ ), as well as team performance (Howell and Avolio, 1993).

Different studies found relationships between HRM practices and organizational goals' achievement (e.g.
Boselie, Paauwe, and Jansen, 2000; Delery and Doty, 1996; West, 1997) and companies' capacity to innovate (Song, Yaping, and Cass 2011). Organizational outcomes require employees' commitment that in turn needs a coherent and consistent set of HR practices to be reached (Walton, 1985). Commitment-oriented HRM practices would promote desired attitudes and behaviors from employees through setting psychological ties between individual and organizational goals. These practices will be more able to produce employees with behaviors prone to organizational aims (Organ, 1988), beyond specific job requirement and despite explicit rewards (Arthur, 1994). Employees interpret HRM as the social cues of organizations' commitment towards their staff (Whitener, 2001), and so they reciprocate with their own commitment to the company.

The scarce amount of research about the impact of HRM practices on organizational climate and culture (Rogg, Schmidt, Shull, and Schmitt, 2001) showed relationship between downsizing practices and negative overall climate perceptions (Luthans and Sommer, 1999), and training opportunities with organizational commitment (Tesluk, Farr, Mathieu, and Vance, 1995). HRM practices could promote an organizational climate that is positive regarding organizational goals. Training information and rewards act as signals that transmit to employees the importance of organizational goals.

So, leadership and HRM practices are means used by organizations to shape perceptions about social environment, giving support to organizational innovation. Leaders provide interpretations that members should accept, influencing their climate perceptions. HRM practices and policies give information about organizational goals, aiming to align individual behaviors with organizational mission (Schneider, Goldstein, and Smith, 1995). From this framework, this study analyzes the relationship between leadership and HRM as antecedents of organizational climate of innovation. Transformational dimensions of leadership are expected as main predictors of innovative climate, mainly inspiration. Commitment-oriented HRM practices are expected as significantly related with innovation climate, with practices addressed to gain participation of employees (showing support and confidence, and allowing risk-taking and mistakes if necessary) as stronger effect. Taken together, inspirational leadership and stimulating participation HRM practices are expected to maintain their predictive capacity on innovative climate.

\section{Method}

The sample is composed by 458 workers becoming to 16 Spanish organizations from different sectors as 
transport, energy, consultancy, technology, health care, educations, industrial supplies and food distribution. All companies were private owned, excepting a public hospital. 13 companies have less than 250 employees, and 3 are transnational enterprises with more than 250 employees. Mean age are 36.9 years (50\% are 29-44 years-old), tenure mean value is 10.8 (50\% of workers have between 2 and 18), and gender distribution is $47.6 \%$ men and $46.1 \%$ women. Jobs performed are first-level workers $(14.8 \%)$, administrative $(21.2 \%)$, technicians $(41.7 \%)$, and middle or high managers (15.1\%) (3.3\% performed other jobs). Finally, $57.9 \%$ of surveyed workers have university training.

Innovation climate was measured through the scale of flexibility and innovation from Paterson et al. (2005) Organizational Climate Measure, based on the Quinn and Rohrbaugh's (1983) model of competing values. Six items with a Likert-scale of four answer's options form this dimension (Cronbach's alpha $=.91$ ).

Leadership is measured with the Multifactorial Leadership Questionnaire, form 5x (Bass \& Avolio, 1990), with 36 items defining five transformational dimensions (intellectual stimulation, inspiration, attributed idealized influence-charisma, behavioral idealized influence-charisma, and individual consideration), three transactional leadership (contingent rewards, active management by exception, and passive management by exception, not included in the study because its low reliability index), and one dimension of no leadership (laissez-faire). Each dimension included four items.

The scale developed by Boselie, Hesselink, Paauwe and van der Wiele (2001) was used to measure commitment-oriented HRM practices. The scale includes four dimensions: employees' participation practices (6 items), sharing information practices (4 items), training and development practices (3 items) and rewards and incentives ( 3 items).

To test the relationships among study variables, a series of hierarchical regression analysis were carried out. First, innovation climate was regressed to leadership styles in four steps. First step included the job as control variable, due the differences found in the literature about innovation demands in different jobs and organizational levels. Second step included as predictors the five transformational dimensions. Third model included the two transactional dimensions of leadership, as well as the previously entered variables. Last model included in the fourth step the laissez-faire dimension. Another regression analysis was carried out using as predictors of innovation climate the four types of HRM practices, entering each one step by step, including job status as control variable. Finally, a third regression analysis was carried out, using as predictors of innovation climate the significant predictors of previous analysis, including the leadership dimensions in the first step and HRM practices in the second step.

\section{Results}

After controlling the job performed by employees, leadership styles predict a significant amount of variance in innovation climate (32.2\%). Despite the significant correlations between all leadership dimensions and innovation climate, the higher predictive capacity comes from inspirational motivation, followed by contingent reward and individualized consideration. The rest of leadership styles don't add explained variance when entered in the regression model.

HRM practices also predict a high amount of the innovation climate variance $(56.3 \%)$, after controlling the job performed by respondents. The regression analysis including the four dimensions of HRM showed that practices addressed to stimulate employee's participation are the main predictor of innovation climate, but sharing information practices and training and development practices contribute to explain additional variance.

Significant predictors of innovation climate found in the aforementioned regression analysis were entered together in a regression model. Results showed that HRM practices increase explained variance in $25.1 \%$, beyond the $33.2 \%$ explained by leadership dimensions. But more interestingly, leadership variables lose their predictive capacity on innovation climate when HRM practices enter in the regression model. Only practices addressed to stimulate employee's participation and training and development practices maintain their predictive capacity, being responsible for the most of the $57.8 \%$ of explained variables.

\section{Discussion}

Ekvall (1996) pointed out that innovation climate was the main differential factor among innovative and non-innovative companies. So, it is interesting to know antecedents of innovation climate. This study considers leadership and HRM practices as variables that increase employees' perceptions about innovations in their companies. People perceiving stimulating leadership and commitment-oriented HRM practices will be more oriented and committed to innovate, devoting more efforts to generate, promote and implement innovative changes in their work.

Leadership and HRM practices are important ways of employee-company interaction, used by employees to develop their perceptions about their organizational context, following Attraction-Selection-Attrition model (Schneider, 1987). Moreover, from psychological contract framework, these signals define the individuals' beliefs regarding the reciprocal commitments of employees and companies to the other party (Rousseau, 1990).

Three leadership dimensions significantly predicts innovation climate, with Inspiration as the main pre- 
dictor. Inspirational leaders generate enough self-confidence in employees to defend new ideas and get support for developing them. Pons and Ramos (2009) found inspiration as the main predictor of the more interactive phase from the innovation process, promotion of innovations, but not in the other two phases (generation and implementation of new ideas).

Contingent reward is also related with innovative climate. As social exchange theories state, distribution and exchange of good and resources in organizations were based on collective and reciprocal behaviors (Fernández-Ríos, 1999). From this view, perception of innovative climate could arise from equity (or inequity) perceptions, which in turn rests on social exchange process derived from interpersonal relations and interaction with the company and direct supervisor.

Individualized consideration added explained variance of innovation climate. Leaders considering individual needs help to develop subordinates' self-confidence, empowering individuals to perform beyond their formal requirements (Fuller, Marler, and Hester, 2006). As in the case of inspiration, perception of supervisor's support promotes the perceptions of climate as facilitating innovations through the level of trust emerged in employees (Seers, 1989).

As leadership, HRM practices showed significant relationships with innovative climate. Three types of practices were significant predictors of innovation climate and only reward practices are not related with this variable. Moreover, HRM practices reduced the predictive capacity of leadership styles when both variables enter the same regression model (only inspiration remains near the level of statistical significance, $\beta .086$, $\mathrm{p}=0.88$ ). These relationships reinforce Whitener (2001) suggestion about employees' interpretation of HRM practices as signals of company commitment, creating perceptions of an organizational climate that facilitates innovation and commitment (Mowday, Steers, and Porter, 1979).

HRM practices devoted to stimulate members' participation showed the higher capacity power on innovative climate. People link their perceptions of company as stimulating participation with opportunities to innovate in the work place, including the allowance of assuming risks and having mistakes. Training and development practices added explained variance on innovative climate and seems to affect the employees' perception about company orientation to innovate, in congruence with previous studies (e.g., Delaney and Huselid, 1996; Harel and Tzafrir, 1999) which found training effects on perceive organizational performance. Relationship between sharing information practices an innovative climate are near statistical significance. Access to information transmits confidence to employees, improving their decisions quality (Vlachos, 2009). Sharing information allows individual promotion through the help to develop new organizational functions (Burguess, 2005).
In sum, obtained results confirm that leadership styles (inspiration, contingent rewards and individualized consideration) contribute to develop employees' perceptions of innovative climate in organizations. Beyond this relationship, when companies develop HRM practices devoted to stimulate members' participation and empowering workers, employees perceive climate as more innovative. Leadership is important to configure organizational climate as innovative, but HRM practices appears as the critical factor if companies want to promote an orientation to innovate. Stimulating participation from employees, giving opportunities to training and development, and in lesser extent, sharing information practices and inspirational leadership appears as effective tools for creating an innovative climate in organizations, which in turn, will lead to more innovative employees.

\section{Referencias}

Abbey. A., \& Dickson, J. (1983). R \& D work climate and innovation in semiconductors. Academy of Management Journal, 26, 362-368.

Amabile, T. M. (1983). The social psychology of creativity. New York: Springer-Verlag.

Amabile, T. M. (1988). A model of creativity and innovation in organizations. Research in Organizational Behaviour, 10, 123-167.

Amabile, T. M., \& Gryskiewicz, N. D. (1989). The creative environment scales: Work Environment Inventory. Creativity Research Journal, 2, 231-253.

Amabile, T. M., Taylor, S., \& Gryskiewicz, N. D. (1995). Technical manual for KEYS: Assessing the Climate for Creativity. Greensboro, NC: Centre for Creative Leadership.

Anderson, N. R., De Dreu, C., \& Nijstad, B. (2004). The routinization of innovation research: a constructively critical review of the state-of-the-science. Journal of Organizational Behavior, 25, 147-173.

Anderson, N. R., \& West, M. A. (1992). Team climates for innovation. Memo $\mathrm{n}^{\circ}$. 1430. MRC/ESRC Social and applied Psychology Unit, University of Sheffield, UK.

Angle, H. (1989). Psychology and organizational innovation. En A. Van de Ven, H. Angle y M. Poole (Eds.). Research on the management of innovation: The Minnesota studies, 135-170. New York: Harper \& Row.

Arthur, J. B. (1994). Effects of Human Resource Systems on Manufacturing Performance and Turnover. Academy of Management Journal, 3, 670-687.

Avolio, B. J. (1999). Full leadership development: Building the vital forces in organizations. Thousand Oaks, CA: Sage.

Avolio, B. J., \& Bass, B. M. (1991). The full range of leadership development: Basic and advanced manuals. Binghamton: Bass, Avolio \& Associates.

Axtell, C. M., Holman, D. J., Unsworth, K. L., Wall, T. D., Waterson, P. E., \& Harrington, E. (2000). Shopfloor innovation: Facilitating the suggestion and implementation of 
ideas. Journal of Occupational and Organizational Psychology, 73, 265-286.

Baer, M., \& Frese, M. (2003). Innovation is not enough: Climates for initiative and psychological safety, process innovations and firm performance. Journal of Organizational Behavior, 24, 45-68.

Bandura, A. (1988). Self-regulation of motivation and action through goal systems. En V. Hamilton \& G. H. Bower (Eds): Cognitive perspectives on emotion and motivation. NATO ASI series D: Behavioral and social sciences, 44, 37-61. Dordrecht, Netherlands: Kluwer Academic Publishers.

Bass, B. M. (1985). Leadership and performance beyond expectations. New York: Free Press.

Bass, B. M. (1998). Transformational leadership: Industry, military, and educational impact. Mahwah, NJ: Lawrence Erlbaum.

Bass, B. M. (1999). Two decades of research and development in Transformational Leadership. European Journal of Work and Organizational Psychology, 8, 9-32.

Bass, B. M., \& Avolio, B. J. (1990). Transformational leadership development: Manual for the Multifactor Leadership Questionnaire. Palo Alto, CA: Consulting Psychologist Press.

Bass, B. M., \& Avolio, B. J. (1994). Shatter the Glass Ceiling: Women May Make Better Managers. Human Resource Management, 3, 549-560.

Bassett-Jones, N. (2005). The Paradox of Diversity Management, Creativity and Innovation. Diversity Management, Creativity and Innovation, 14, 169-175.

Becker, B. E., \& Gerhart, B. (1996). The impact of human resource management on organizational performance: progress and prospects. Academy of Management Journal, 39, 779-801.

Boselie, P., Hesselink, M., Paauwe, J., \& van der Wiele, T. (2001). Employee perceptions on commitment orientated work systems. Rotterdam, ERIM Workpaper.

Boselie, P., Paauwe, J., \& Jansen, P. G. W. (2000). Human Resource Management \& Performance: lessons from the Netherlands, invited paper IIRA 12th World Congress, Tokyo, Japan.

Burgess, D. (2005). What motivates employees to transfer knowledge outside their work unit? Journal of Business Communication, 42, 324-348.

Butler, J. E., Ferris, G. R., \& Napier, N. K. (1991). Strategy and human resources management. Cincinnati: SouthWestern.

Chang, S., Gong, Y., \& Shum, C. (2011). Promoting innovation in hospitality companies through human resource management practices. International Journal of Hospitality Management, 30, 812-818.

Charbonnier-Voirin, A., El Akremi, A., \& Vandenberghe, C. (2010). A Multilevel model of transformational leadership and adaptive performance and the moderating role of climate for innovation. Group Organization Management, 35, 699-726.

Chesnais, F. (1990). Science, technologie et compétitivité. STI Revue, 1, Paris
Chow, C. W., Harrison, G. L., McKinnon, J. L., \& Wu, A. (1999). Cultural influences on informal information sharing in Chinese and Anglo-American organizations: An exploratory study. Accounting, Organizations and Society, 24, 561-582.

Delaney, J. T., \& Huselid, M. (1996). The impact of human resource practices on perceptions of organizational performance. Academy of Management Journal, 39, 949969.

Delaney, J. T, Lewin, D., \& Ichniowski, C. (1989). Human resource policies and practices in American firms. Washington: Government Printing Office.

Delery, J. E., \& Doty, D. H. (1996). Modes of Theorizing in Strategic Human Resource Management: tests of universalistic, contingency, and configurational performance predictions. Academy of Management Journal, 4, 802-35.

De Dreu, C. K. W., \& De Vries, N. K. (1997). Minority dissent in organizations. En C. K. W. De Dreu y E. Van de Vliert (Eds.). Using conflict in organizations, 72-86. London: Sage.

De Dreu, C .K. W., \& West, M. A. (2001). Minority dissent and team innovation: the importance of participation in decision making. Journal of Applied Psychology, 86, 1191-1201.

Dorenbosch, L., Van Engen, M., \& Verhagen, M. (2005). Onthe-job Innovation: The Impact of Job Design and Human Resource Management through Production Ownership. Creativity and Innovation Management, 14, 129-141.

Doty, D. H., \& Glick, W. H (1994). Typologies as a unique form of theory building: Toward improved understanding and modeling. Academy of Management Review, 19, 230251.

Donati, P. (1997). El desarrollo de las organizaciones del Tercer Sector en el proceso de modernización y más allá. Revista Española de Investigaciones Sociológicas, 79, 113-142.

Dyer, L. (1985). Strategic human resources management and planning. In K. Rowland \& G. Ferris (Eds.): Research in Personnel and Human Resource Management, 3, 1-30. Greenwich, CT: JAI Press.

Eisenbeiss, S. A., van Knippenberg, D., \& Boerner, S. (2008). Transformational leadership and team innovation: Integrating team climate principles. Journal of Applied Psychology, 93, 1438-1446.

Ekvall, G. (1996). Organizational climate for creativity and innovation. European Journal of Work and Organizational Psychology, 5, 105-123.

Elenkov, D., \& Manev, I. (2005). Top management leadership and influence on innovation: The role of sociocultural context. Journal of Management, 31, 381-402.

Fernández-Ríos, M. (1999). Diccionario de Recursos Humanos: Organización y Dirección. Madrid: Ed. Díaz de Santos.

Fernie, S., Metcalf, D., \& Woodland, S. (1995). Corrosion of Collective Industrial Relations: Causes and Consequences. Paper presented at the Seminar HRM \& Performance, Septiembre. Rotterdam: Erasmus University. 
Fombrun, C., Tichy, N., \& Devanna, M. (1994). Strategic human resource management. New York: Wiley.

Ford. C. M., \& Gioia, D. A. (Eds.). (1995). Creative action in organizations: Ivory tower visions and real world voices. Thousand Oaks, CA: London Sage.

Fuller, J. B., Marler, L. E., \& Hester, K. (2006). Promoting felt responsibility for constructive change and proactive behavior: Exploring aspects of an elaborated model of work design. Journal of Organizational Behavior, 27, 1089-1120.

Garrahan, P., \& Stewart, P. (1992). The Nissan enigma: Flexibility at work in a local economy. London: Mansell.

Gil-Monte, P.R. (2001). El síndrome de quemarse por el trabajo (sindrome de burnout): aproximaciones teóricas para su explicación y recomendaciones para la intervención. Psycologia.com.

Glick, W. H. (1985). Conceptualizing and measuring organizational and psychological climate: pitfall in multilevel research. Academy of Management Review, 10, 601-616.

Glick, W. H. (1988). Response: organizations are not central tendencies. Shadowboxing in the dark, round 2. Academy of Management Review, 13, 133-137.

Golden, K., \& Ramanujan, V. (1985). Between a dream and a nightmare: On the integration of the human resource management and strategic business planning processes. Human Resource Management, 24, 429-452.

Harel, H. G., \& Tzafrir, S. S. (1999). The effect of human resource management practices on the perceptions of organizational and market performance of the firm. Human Resource Management, 38, 185-200.

Hater, J. J., \& Bass, B. M. (1988). Supervisors' evaluations and subordinates' perceptions of Transformational and Transactional Leadership. Journal of Applied Psychology, 73, 695-702.

Howell, J. M., \& Avolio, B. J. (1993). Transformational leadership, transactional leadership, locus of control, and support for innovations: Key predictors of consolidated-business-unit performance. Journal of Applied Psychology, 78, 891-903.

Huselid, M. (1993). Estimates of the impact of human resource management practices on turnover and productivity. Paper presented at the Academy of Management Annual Conference.

Huselid, M. (1995). The impact of human resource management practices on turnover, product corporate financial performance. Academy of Management Journal, 38, 635672.

Isaksen, S. G., Lauer, K .J., Ekvall, G., \& Britz, A. (20002001) Perceptions of the best and worst climates for creativity: Preliminary validation evidence for situational outlook questionnaire. Creativity Research Jour-nal, 13, 171-184.

James, L. R., Hartman, A., Stebbins, M., \& Jones, A.P. (1977). Relationships between psychological climate and a VIE model for work motivation. Personnel Psychology, 30, 229-254.

James, L. R, James, L., \& Ashe (1990). The meaning of organizations: the role of cognition and values. In B.
Schneider (Ed.): Organizational climate and culture, 4084. San Francisco: Jossey-Bass.

James, L. R., \& Sells, S. (1981). Psychological climate: Theoretical perspectives and empirical research. In D. Magnussen (Ed.): Toward a psychology of situations: An interactional perspective, 275-295. Hillsdale, NJ: Erlbaum.

Janssen, O. (2005). The joint impact of perceived influence and supervisor supportiveness on employee innovative behaviour. Journal of Occupational and Organizational Psychology, 78, 573-579.

Janssen, O., De Vliert, E., \& West, M. (2004). The bright and dark sides of individual and group innovation: a Special Issue introduction. Journal of Organizational Behavior, $25,129-145$

Janssen, O., Schoonebeek, G., \& van Looy, B. (1997). Cognities van empowerment als de schakel tussen delegerend leiderschap en innovatief gedrag van werknemers. Gedrag en Organisatie, 10, 175-194.

Jung, D., Chow, C. W., \& Wu, A. (2003). The role of transformational leadership in enhancing organizational innovation: Hypotheses and some preliminary findings. Leadership Quarterly, 14, 525-544.

Kalleberg, A., \& Moody, J. (1994). Human Resource Management and Organizational Performance. American Behavioral Scientist, 7, 948-962.

Kanter, R. (1983). The change masters. New York: Simon \& Schuster.

King, N., \& Anderson, N. (1995). Innovation and change in organizations. London: Routledge.

King, N., \& Anderson, N. (2002). Managing innovation and change: A critical guide for organizations. London: Thomson.

Kisfalvi, V., \& Pitcher, P. (2003). Doing what feels right: The influence of CEO character and emotions on top management team dynamics. Journal of Management Inquiry, $12,42-66$

Kuenzi, M., \& Schminke, M. (2009). Assembling fragments into a lens: A review, critique, and proposed research agenda for the organizational work climate literature. Journal of Management, 35, 634-717.

Kwasniewska, J., \& Necka, E. (2004). Perception of the Climate for Creativity in the Workplace: the Role of the Level in the Organization and Gender. Creativity and Innovation Management, 13, 187-196.

Luthans, B. C., \& Sommer, S.M. (1999). The impact of downsizing on workplace attitudes. Group and Organization Management, 24, 46-70.

Mañas, M. A., González, V. , \& Peiró, J. M. (1999). El clima de los equipos de trabajo: determinantes y consecuencias. Almería: Universidad de Almería (Ed.). Monografías Humanidades 22.

Moriones, A., Merino, J., \& Cerio, D. (2002). Las prácticas de recursos humanos de alto compromiso: un estudio de los factores que inciden en su adopción en la industria española. Cuadernos de Economía y Dirección de la Empresa, 12, 227-246.

Morishima, M. (1991). Information sharing and firm performance in Japan. Industrial Relations, 30, 37-61. 
Moss, S. A., Dowling, N., \& Callanan, J. (2009). Towards an integrated model of leadership and self regulation. Leadership Quarterly, 20, 162-176.

Mowday, R. T., Steers, R. M., \& Porter, L.W. (1979). Employee-organization linkages: the Psychology of commitment, absenteeism, and turnover. New York: Academic Press.

Mumford, M., Scott, G. M., Gaddis, B., \& Strange, J. M. (2002). Leading creative people: Orchestrating expertise and relationships. Leadership Quarterly, 13, 705-720.

Nicholson, N., \& West, M. (1988). Managerial job change: Men and women in transition. Cambridge: Cambridge University Press.

Organ, D. (1998). Organizational citizenship behavior. The good soldier syndrome. Lexington, M. A.: Lexington books.

Osterman, P. (1994). How common is workplace transformation and who adopts it? Industrial and labor relations review, 47, 173-188.

Paolillo, J., \& Brown, W. (1978). How organizational factors affect R \& D innovation. Research Management, 21, 1215.

Patterson, M. G., West, M. A., Shackleton, V. J., Dawson, J. F., Lawthom, R., Maitlis, S. ... Wallace, A. M. (2005). Validating the organizational climate measure: Links to managerial practices, productivity and innovation. Journal of Organizational Behavior, 26, 379-408.

Pfeffer, J. (1994). Competitive advantage through people. California Management Review, 36, 9-28.

Pons, F. J. (2009). Variables psicológicas y organizacionales relacionadas con la conducta de innovación en las organizaciones. Tesis doctoral. Universitat de València.

Quinn, R. E. y Rohrbaugh, J. (1983). A spatial model of effectiveness criteria: toward a competing values approach to organizational analysis. Management Science, 29, 363377.

Rank, J., Nelson, N. E., \& Xu, X. (2004). Predicting innovation: Synergies between leadership and self-related variables. Paper presented at the Annual Meeting of SIOP, Chicago, Illinois, April.

Rogg, K., Schmidt, D., Shull, C., \& Schmitt, N. (2001). Human resource practices, organizational climate, and customer satisfaction. Journal of Management, 27, 431449.

Rousseau, D. (1990). Quantitative assessment of organizational culture: the case for multiple measures. In B. Schneider (Ed.): Frontiers in Industrial and Organizational Psychology, 3, 153-192. San Francisco: Jossey Bass.

Sánchez, I. (2003). Prácticas de recursos humanos y eficacia organizativa: ¿cómo se combinan? Iberoamerican Academy of Management.

Schneider, B. (1975). Organizational climates: An essay. Personnel Psychology. 28, 447-479.

Schneider, B. (1987). The people make the place. Personnel Psychology, 40, 437-454.

Schneider, B. (1990). The climate for service: an application of the climate construct. In B. Schneider (Ed.): Orga- nizational climate and culture, 383-412. San Francisco, CA: Jossey-Bass.

Schneider, B. (2000). The psychological life of organizations. In N. M. Ashkanasy, C. P. M. Wilderon \& M. F. Peterson (Eds.): Handbook of organizational culture and climate, xvii-xxi. Thousand Oaks, CA: Sage.

Schneider, B., Goldstein, H. W., \& Smith, D. B. (1995). The ASA framework: an update. Personnel Psychology, 48, $747-773$

Schneider, B., \& Reichers, A. (1983). On the etiology of climates. Personnel Psychology, 36, 19-39.

Scott, S., \& Bruce, R. (1994). Determinants of innovative behaviour: a path model of individual innovation in the workplace. Academy of Management Journal, 37, 580607.

Seers, A. (1989). Team-Member Exchange quality: A new construct for role-making research. Organizational Behavior and Human Decision Processes, 43, 118-135.

Siegel, S., \& Kaemmerer, W. (1978). Measuring the perceived support for innovation in organizations. Journal of Applied Psychology, 63, 553-562.

Song, C., Yaping, G., \& Cass, S. (2011). Promoting innovation in hospitality companies through human resource management practices. International Journal of Hospitality Management, 30, 812-818.

Stoker, J. I., Looise, J. C., Fisscher, O. A. M., \& de Jong, R. D. (2001). Leadership and innovation: relations between leadership, individual characteristics and the functioning of $\mathrm{R} \& \mathrm{D}$ teams. International Journal of Human Resource Management, 12, 1141-1151.

Terpstra, D. E., \& Rozell, E. J. (1994). The relationship of staffing practices to organizational level in performance. Personnel Psychology, 46, 27-48.

Tesluk, P. E., Farr, J. L., Mathieu, J. E., \& Vance, R. J. (1995). Generalization of employee involvement training to the job setting: individual and situational effects. Personnel Psychology, 48, 607-632.

Tjosvold, D. (1998). The cooperative and competitive goal approach to conflict: accomplishments and challenges. Applied Psychology: An International Review, 47, 285313.

Van de Ven, A., Angle, H. L., \& Poole, M. (Eds.). (1989). Research on the management of innovation: The Minnesota studies. New York: Harper \& Row.

Van Knippenberg, D., \& Ellemers, N. (2004). Social identity and group performance. Identification as the key to Group-Oriented Effort. In A. Haslam, D. V. Knippenberg, M. J. Platow \& N. Ellemers (Eds.): Social identity at work. Developing theory for organizational practices, 2959. New York: Psychology Press.

Vlachos, I. P. (2009). The effects of human resource practices on firm growth. International Journal of Business Science and Applied Management, 4, 17-34.

Wallace, J. E. (1995) Corporatist control and organizational commitment among professionals: The case of lawyers working in law firms. Social Forces, 3, 811-40.

Walton, R. A. (1985). From control to commitment in the workplace. Harvard Business Review, 63, 77-84. 
West, M. A. (1990). The social psychology of innovation in groups. In M. A. West \& J. L. Farr (Eds.): Innovation and creativity at work: Psychological and organizational strategies. Chichester: Wiley.

West, M. A. (1997). Developing creativity in organizations. Leicester: BPS Books.

West, M. A. (2002). Sparkling fountains or stagnant ponds: An integrative model of creativity and innovation in work groups. Applied Psychology: An International Review, $51,355-386$.

West, M. A., \& Anderson, N. (1996). Innovation in top management teams. Journal of Applied Psychology, 81, 680-693.
West, M. A., \& Farr. J. (1989). Innovation at work: Psychological perspectives. Social Behavior, 4, 1530 .

West, M. A., \& Farr. J. (1990). Innovation and creativity at work: Psychological and Organizational Strategies. Chichester: Willey.

West, M. A., \& Wallace, M. (1991). Innovation in health care teams. European Journal of Social Psychology, 21, 303315.

Whitener, E. M. (2001). Do 'high commitment' human resource practices affect employee commitment? Journal of Management, 27, 515-535.

Manuscrito Recibido: 23/04/2012

Revisión Recibida: 14/08/2012

Aceptado: 14/08/2012 